\title{
The Up-to-Date Progression and Prospect Drift of Wireless Sensor Networks
}

\author{
Hathal Salamah A. Alwageed \\ Department of Computer Engineering and Network \\ Aljouf University, Saudi Arabia
}

\begin{abstract}
WSN's have transformed into a champion amongst the most interesting scopes of investigation in the past couple of years. It made out of different wireless sensor nodes which outline a sensor field and a sink. These tremendous amounts of nodes, having the abilities to sense their surroundings, perform limited retribution and confer remotely shape the wireless sensor networks. Late advances in remote and electronic headways have engaged a far reaching mixed bag of exercises of wireless sensor networks in military, movement observation, Traffic, focus taking after, environment watching, healthcare, and whatnot. There are various new challenges that have surfaced for the originators of wireless sensor networks with a particular finished objective to meet the requirements of distinctive applications like identified sums, size of nodes, and its self-sufficiency. Hence, updates in the present developments and better responses for these challenges are required. The future enhancements in sensor nodes must convey successful and economical contraptions, so that they may be brought into play as a piece of usage like submerged acoustic sensor systems, distinguishing based cyber-physical structures, time-critical applications, cognitive recognizing and management of spectrum, and security and assurance services.
\end{abstract}

\section{Keywords}

WSN's, Senor Nodes, Communication, Trends, Development

\section{INTRODUCTION}

WSNs have moreover evolved with the advances in the development of micro-electromechanical system (MEMS), progressions in remote correspondences (Wireless Communication). Wireless sensor networks have transformed into a champion amongst the most intriguing domains of examination in the past couple of years. Wireless sensor networks are commonly made out of little, economical contraptions or device that bestows remotely and have the limits of taking care of, identifying and securing. The change of Wireless sensor networks was impelled by military applications, for instance, battle zone perception. WSN are being brought into play as a piece of various industrial and normal national application zones, taking in Industrial process monitoring and control portrayed by [1] healthcare observations depicted by [2] environment and domain watching, restorative administrations applications, home computerization, and development control displayed well by[1][3]. A Wireless sensor network overall entails a base station that can communicate with different sensors by method of a radio association/link. Sensor nodes accumulate the data, pack or compress it, and transmit it to the gateway particularly or by suggestion with the support of diverse nodes. The transmitted data is then acquainted with the system by the gateway connection. An analysis of the late advances in Wireless sensor networks has been outlined here that enable a far reaching assortment of employments and future change in applications like submerged acoustic sensor systems; recognizing based cyber- physical systems, time-critical applications etc.

\section{UP-TO-DATE PROGRESSION}

Up-to-date progression in wireless and electronic headways/technologies has engaged a far reaching mixture of employments of Wireless sensor networks in diverse fields.

\subsection{Sensor Localization and \\ Location-Aware Services}

\subsubsection{Smart Home}

Such state of affairs can bestow custom practices to a given individual. Far reaching measure of investigation has been given to this point. The examination on smart homes is presently starting to progress into the business area. It takes a considerable measure of work and needing to make a sagacious home. There are various instances of things at this moment accessible which can perform singular limits that are thought to be a bit of a smart home. A couple of important applications which misuse information assembled by Wireless sensor networks are shown by [4].

\subsubsection{Monitoring and Management of Traffic}

Every tremendous city is encountering traffic deadlocks or congestion the world over. A sincere effort is being made to handle it. Such problems can be helped by orchestrating supervising traffic. A real-time customized traffic data amassing must be brought into play for capable organization of rush-hour action. Research on this subject is considered as a highlight of the Intelligent Transport System (ITS) exploration bunch. [5] uncovered ITS to be the utilization of the PCs, correspondences, and sensor advancement to surface transportation. The vehicle taking after or tracking application is to locate a specific vehicle or moving dissent and screen its behavior. This work moreover depicts design of Wireless sensor network for vehicular checking. As the power source (battery) is restricted, it is discriminating that a framework/network of sensor nodes is power gifted.

\subsubsection{Military}

New and creating advancements, for instance, frameworks or networks, reinforce military operations by passing on essential information rapidly and continually to the other side individual or relationship at the right time. This improves the capability of fight operations. The new developments must be fused quickly into a complete basic designing to meet the requirements of present time. Change in conditions care or awareness [6] is must essential. [7] portrayed some other discriminating application is disclosure of enemy units' improvements aground/sea, recognizing intruders on bases, 
chemical or biological risks and offering logistics in urban battling. Command, control, correspondences, figuring, information, perception, observation, and concentrating on structures are all that much depicted by [8].

\subsubsection{Healthcare}

Structures are examined at general time breaks, and repairing or supplanting in light of the season of usage, rather than on their working conditions. [9] has elucidated that sensors embedded into structures engage condition-based upkeep of these favorable circumstances. Wireless sensing will allow assets for be explored when the sensors exhibit that there may be an issue. This will trim down the outlay of upkeep and foreseeing harmful disillusionment. These applications fuse sensors mounted on generous commitment compasses, inside concrete and composite materials [10], and tremendous structures.

\subsubsection{Commercial and Industrialized}

Since the long time remote/wireless transmission of data is being done in industrial applications, however starting late it has gotten essentialness. Productive draw on of wireless sensors in structures, for instance, SCADA has exhibited that these devices could reasonably address the needs of present day applications. The fundamental strategy employments of Wireless sensor networks in industry are watching temperature, flow-level, and pressure parameters. With the rapidly growing progression in the technology of wireless and its subsequently lessening expenses, different remote applications are being made in industry. Wireless sensor network in gathering business undertakings can screen and development quality control.

\subsubsection{Fostering/Agriculture}

[11] Communicated that agriculture can moreover be benefitted by the sending of Wireless sensor network to get the information regarding soil degradation and water deficiency. With help of Wireless sensor networks we can test out the clean water ate up in watering framework/network and administer it.

\subsection{Coverage and Topology Control}

Topology control is one of the focal issues in Wireless sensor networks. It has great essentialness for postponement lifetime, diminishing radio impediment, extending the adequacy of media access control and routing protocols. It furthermore guarantees the coverage and connectivity quality and increase in the service of framework/network as well. An enormous propel in investigation can be seen in Wireless sensor networks topology control. Various topology control estimations have been delivered till date, yet issues, for instance, unlucky deficiency of clear and rational algorithms, nonappearance of gainful estimation of framework/network execution and profitable nature of numerical model still exist. A couple outline/graph models brought into play as a piece of topology control, the present issue ranges and the future examples on the investigation of topology control are presented by [12]

\subsubsection{Quality of Service (QoS)}

QoS support is attempting on account of compelling energy and computational resource propels of wireless sensors. Distinctive service properties, for instance, the deferment or delay, resolute quality or reliability, framework/network lifetime, and data quality may conflict; For case, multi-path routing can improve the reliability; at any rate it can grow the energy usage and concede as a result of duplicate transmissions. Showing such associations, measuring the bestowed quality, and offering expects to control the adjustment is central for QoS hold up. There are distinctive examination opportunities in enhancing the QoSof Wireless sensor networks. One of the looks at is the endeavor delineated by [13] that separates and enhances the execution of a Wireless sensor network by taking in an essential max-min fairness bandwidth allocation approach.

\subsubsection{Mobility}

It is a champion amongst the most crucial issues in front line frameworks/networks. As Wireless sensor networks are transforming into the accompanying parts without limits Internet, it is basic to study new-fangled models that furthermore reinforce adaptability of these nodes. Wireless sensor networks are suitable in blended sack of cases that make it difficult to convey a standard compactness circumstance. Taking after are a couple of circumstances where the convenient support is indispensable offered in [14] Intra-WSN contraption/device improvement is likely the most surely understood circumstance in Wireless sensor networks architectures, where each sensor node can alter from its neighborhood position at run time devoid of losing the system with the sensor switch (SR). Because of inter-WSN device improvement, sensor nodes stir between particular sensors composes each one with its SR tried and true to plan and manage all the collected devices. An investigation errand of IETF working social affair NEMO, an instance of WSN advancement is portrayed in RFC-3963 by [15]. Sensor framework/network passed on in a moving transport is a bona fide circumstance of this sort. It is possible to have a circumstance where a sensor network can employ another sensor compose to be related through Internet. MANEMO [16] endeavor is also a representation.

\subsubsection{Privacy and Security Apprehension}

The field that gave cautious thought is the security distress on information being accumulated, transmitted, and separated in a Wireless sensor network. Such private information of concern may consolidate payload data accumulated by sensors and transmitted through the framework/network to a centralized data get ready server. The territory of a sensor beginning data correspondence, and other such association information, may similarly be the focal point of assurance concerns. In bona fide employments of Wireless sensor networks, fruitful countermeasures against the introduction of both data and context-oriented private information are fundamental essentials. Security in distinctive fields related to Wireless sensor networks, for instance, wired and wireless networking, databases and data-mining, has been extensively thought about by [17]. Convincing privacy-preserving frameworks/networks are needed for the exceptional challenges of Wireless sensor networks safety measures.

\subsubsection{Biomedical/Medical}

The jobs of Wireless sensor networks in medical field are in creating stage. Biomedical wireless sensor networks (BWSNs) show the prospect open entryways for supporting flexibility while watching key body lives up to expectations in hospital and home care. There is a need for BWSN to develop in order to cover security dealing with, improved sign compromise and visualization. They can in like manner be brought into play to accomplish increased compactness outside the surgery room, seeing of a couple of patients/persons meanwhile, and further changes in 
accordance with therapeutic expert's prerequisites for information. As the Internet utilization has been able to be popular among people, e-services for the healthcare which is normally known as e-Health, have starting late pulled in huge thought inside both the investigation society and industry. Followings are a couple of persistent exercises for restorative administrations utilizing Wireless sensor networks:

- CodeBlue [18] - a development displaying planned for taking after and checking of patients.

- $\quad$ ALARM-NET [19] - a Wireless sensor networks produced for assisted-living and private checking.

- AMON [20] - a Wireless Body Area Sensor Network System

- GlucoWatch G2 [21] - bring into play Wireless sensor networks to research wearable individual healthcare system that will screen and survey human urgent signs.

\section{PROSPECT DRIFT}

The upcoming changes in sensor nodes have got to convey able and economical contraptions or devices, so that they may be utilized as a piece of applications like underwater acoustic sensor systems, sensing based cyber-physical structures/systems, time-critical applications, cognitive sensing etc.

\subsection{Submerged or underwater Acoustic Sensor Systems}

[22] Displayed a complete study in such networks. They are premeditated to engage applications for oceanographic data aggregation, sullying checking, toward the ocean examination, disaster suspicion, upheld course and key surveillance applications. These sensors are moreover being utilized for examination of general undersea resources and get-together of investigative data. So a need of submerged/underwater correspondences among submerged or underwater contraptions rises. These sensor nodes and vehicles should be fit for bearing their operation, exchanging their territory and improvement information and thus relay watched data to a seaside base-station. Another investigation standard UWSNs positions challenges like tremendous inducing delays, node flexibility or mobility and high slip by probability of acoustic underwater channels, appeared differently in relation to the ground-based wireless sensor networks. A GPS free routing Protocol named DUCS (Distributed Underwater Clustering Scheme) portrayed by [23]. It lessens the proactive or hands-on routing message exchange and does not bring into play flooding approaches. It in like manner employs data collection to discard dreary information.

\subsection{Cognitive Sensing}

These sensor networks are brought into play for getting limited and orchestrated information of the recognizing/sensing environment by the sending an immense sensors splendidly and autonomically. Managing a far reaching number of remote sensors is a baffling errand. As [24] portrayed, a critical examination distraction can be seen in bio-inspired sensing and networking. Two doubtlessly comprehended representations of subjective distinguishing are Quorum sensing and swarm intelligence:

a. Quorum sensing is an example of bio-inspired sensing and networking. Dominant part recognizing is the limit of microorganisms to pass on and coordinate direct by method of signaling molecules.

b. Swarm intelligence is delivered in A.I aptitude for considering the total behavior of decentralized, self-organized structures.

\subsection{Spectrum Management}

As usage of low-power wireless protocols is growing, we can predict an outlook in which remote/wireless devices, for instance, wireless supports, power-point mediators, cell telephone headsets, and healthcare watching sensors will be pervasive. In the meantime, the pervasiveness of these contraptions prompts extended impedance and blockage inside and between frameworks/networks, because of covering physical frequencies. Subjective radios and multi-frequency MACs are a few systems that have been delivered to utilize different frequencies for parallel correspondence. A non particular course of action is given by [25] as SAS: a Self-Adaptive Spectrum Management middleware for wireless sensor networks, which can be smoothly consolidated with a present single frequency.

\subsection{Heterogeneous Networks Harmonization}

In view of the fact that the sensor notes are energy restricted so the key hindrance in the coordination/harmonization with diverse networks is obliged energy of sensor nodes. Sensor networks are amazingly important for applications like healthcare watching, wildlife observation, fire detection and building controls. To screen the wireless sensor networks, the data made by sensor nodes should be accessible. This could be conceivable by joining the wireless sensor networks with existing framework/network base, for instance, overall Internet, an area or private web. [26] depicted two kind of interconnection procedures: gateway based interconnection methodology and overlay based interconnection methodology.

\subsection{Time-Critical Applications}

Another time of circled embedded structures, with a wide extent of real-time applications, for instance, border surveillance, fire monitoring, healthcare, and so on, can be addressed by Wireless Sensor Networks. On account of compelling resource constrains in significantly dynamic circumstances these systems face new sorts of timing constraints. Various built approaches to manage real-time preparing like Wireless Sensor Networks protocols, middleware services O.S, data management, programming models, and theoretical examination are tried by Wireless Sensor Networks. The blueprint of complex time-critical structures consolidates particular sorts of information and correspondence advancement systems, for instance, wireless (mesh) sensor networks, to finish control frames consistently.

\subsection{Trial arrangement and new-fangled Applications}

There are giant opportunities to send Wireless Sensor Networks for honest to goodness applications and test set-ups. Wireless Sensor Networks are being sent in diverse application circumstances, comprising rural and woods circumstances. [27] laid out a Wireless Sensor Networks based system for flat target (animal) following in the including district of regular life segments. That is fabricated to construct safe courses for animals to cross transportation bases. This is made on the reason out of the IEEE 802.15.4 
standard. In circumstances like fire hazard monitoring, adaptable routing is imperative remembering the deciding objective to ensure protected and advantageous data transport in building getting and putting out fire hazard applications. The routing protocol presented by [28] is a generous emergency-adaptive protocol for emergency circumstances, for instance, building fire risk applications. This protocol moreover handles dynamic emergency circumstances and capacities commendably with the controlling crevice issue. An endeavor name NEURON projected by [29] has been created to reduce the diserse nature of operation and management, and hold up the spread of data inside future Wireless Sensor Networks. This is an energy capable game plan, clustering and routing segment in Wireless Sensor Networks that spotlights on the joining of programmed functionalities in the present strategies. Conventional sensor networks clustering algorithms can't be brought into play for node clustering for coordination of blended media distinguishing and taking care of. A clustering framework proposed by [30] for Wireless Multimedia Sensor Networks (WMSNs) is in light of secured Field of View (FoV). This clustering framework preserve energy and postponements the network lifetime by encouraging the nodes in such a course, to the point that stays far from the overabundance identifying and planning. Sensor networks can be utilized as a noteworthy contraption/device to deal with the fuel and to manufacture the energy adequacy likewise without diminishing the lifestyle quality. Different sensors are interconnected to shape a sensor sort out inside a building. It is hard to send a control system in a building because of the diverse correspondence models. [31] Made a building plan that displays a web services-based strategy that organizes resource propelled sensor nodes with IP-based frameworks/networks.

\subsection{Innovative Models and Structural Designs}

Wireless Sensor Networks is a autonomous arrangement of battery-powered remote sensors that can sense, change, and bestow. We have discussed various specific troubles so far that legitimacy sincere thought. These defies are more like unlucky deficiency of faultless Wireless Sensor Networks building configuration. Some of starting late made architectures/structural designs are outlined here: the environment adaptive and energy resourceful aptitudes be supposed to be integrant for wireless sensor networks architecture. A different leveled structure and sub-facets limits auxiliary arranging model EAWNA is planned by [32] that in like manner has diverse focuses like adaptability, revamp services, environment adaptable and energy competent. Standard network basic arranging diagrams are in perspective of layering philosophy. The thought about the layered development displaying can in like manner be brought into play with sensor framework/networks building configuration arrangement. Cubic and cross-layer (CCL) by [33] is suitable sensor framework architecture designing because of application-specific nature of sensor frameworks/networks. [34] anticipated Wirelessly Accessible Sensor Populations (WASP) undertaking is to develop a consolidated model for realizing applications using wireless sensor networks. In this wander a model has been made to program complete wireless sensor network with everything taken into account rather than programming individual node in light of the clashing behavior of individual nodes.

\subsection{Problems of Holes}

These are one of the troubles in game plan of wireless sensor networks in a considerable domain. Holes generally considered as a correspondence gap among sensor nodes. [35] depicted diverse sorts of holes like Coverage holes Routing holes, Jamming hole, Sink/Black hole, and Worm holes in their assessment exertion.

\subsection{Problem of Time Synchronization}

Time synchronization in wireless sensor networks, is to bring the clocks of the sensor nodes together faultless (standard) clock. To unite these clocks skew and buoy of the nodes clock need to be administered. Standard test for time synchronization in wireless sensor networks is to diagram a light weight, inadequacy tolerant and energy viable protocol to lessen the energy expenditure.

\section{PROSPECTIVE DEFIES}

Power is reliably been a test for wireless sensor networks traces. One of the ways to deal with drag out the framework/network lifetime is to arrange the energy resourceful algorithms and gear that uses control shrewdly. One of the rule challenges is to make simplicity and minor sensor nodes. Current sensor nodes are basically models with respect to these targets. Simplicity of sensor nodes can be accomplished by late and future propel in the fields of MEMS. Security is one of the critical troubles in wireless sensor networks. Most of the strikes that are performed on wireless sensor networks are insertion of false information by bartered nodes inside the networks. Progression of security arrangements for wireless sensor networks moreover goes up against challenges related to oblige environment. Researches in the field of wireless sensor network is proceeding the world over yet in the meantime there is no bound together system and framework/network building configuration, on the most elevated purpose of that differing application can be collected. Protocols need to be made for certifiable issues considering the speculative thoughts and incorporating novel game plans into a complete system-wideprotocol for genuine application. Till date not a lot of exploratory results exist for wireless sensor networks. Each and every innovative application simply gets conviction when it is attempted \& separated fundamentally and results are differentiated and existing arrangements.

\section{CONCLUSIONS}

The natural method for wireless sensor networks makes them deployable in an arrangement of circumstances. They can perhaps be everywhere, on lanes, in our homes and working environments, timberlands, combat areas, calamity struck regions, and impressively submerged in oceans. We outline the application regions where wireless sensor networks have been passed on, for instance, military distinguishing, development perception, focus taking after, environment watching, and human administrations. We furthermore outline the distinctive fields where wireless sensor networks may be passed on within the near future as submerged acoustic sensor systems, recognizing based cyber-physical structures, time-critical applications, scholarly distinguishing and extent organization, and security and insurance organization. These application domains are being examined comprehensively by distinctive people over the business industry and academic domain. 


\section{REFERENCES}

[1] "R. Kay et al", "The Design Space of Wireless Sensor Networks" IEEE Wireless Communications Vol.11.No.6 (2004) pp 54-61.

[2] "A. Tiwari et al", "Energy-efficient wireless sensor network design and implementation for condition-based maintenance" ACM Transactions on Sensor Networks (TOSN) Vol.3.No.1 (2007) pp 1-7.

[3] "S. Hadim et al", "Middleware: middleware challenges and approaches for wireless sensor networks" Distributed Systems Online, IEEE Vol.7.No.3 (2006) pp 1-1.

[4] "S. Hussain et al", "Applications of Wireless Sensor Networks and RFID in a Smart Home Environment" Proceedings of the 2009 Seventh Annual Communication Networks and Services Research Conference (2009) pp 153-157.

[5] "J. Chinrungrueng et al", "A Vehicular Monitoring System with Power-Efficient Wireless Sensor Networks" ITS Telecommunications Proceedings, 6th InternationalConference on (2006) pp 951-954.

[6] "Chien-Chung Shen et al", "Sensor Information Networking Architecture and Applications" IEEE Personal Communications (2001) pp 52-59.

[7] "S. S. Doumit et al", "Self-Organizing and Energy-Efficient Network of Sensors" IEEE (2002) pp $1-6$.

[8] "I. F. Akyildiz et al", "A Survey on Sensor Networks" IEEE Communications Magazine (2002) pp 102-114.

[9] "A. Tiwari et al", "Design \& Implementation of Wireless Sensor Network for Machine Condition Based Maintenance" Int'l Conf. Control, Automation, Robotics, \& Vision (ICARV), Kunming, China, 6-9 Dec. 2004.

[10] "S.W. Arms et al", "Validation of Remotely Powered and Interrogated Sensing Networks for Composite Cure Monitoring" paper presented at the 8th International Conference on Composites Engineering (ICCE/8), August 7-11, 2001.

[11] "N. Wang et al", "Wireless sensors in agriculture and food industry: Recent development and future perspective" Computers and electronics in agriculture Vol.50.No.1 (2006) pp 1-14.

[12] "S. Jardosh et al", "A Survey: Topology Control for Wireless Sensor Networks" Signal Processing, Communications and Networking, 2008. ICSCN '08. International Conference on, (2008) pp 422-427.

[13] "H. Z. Abidin et al", "Provisioning QoS in Wireless Sensor Networks using a Simple Max-Min Fair Bandwidth Allocation" 2009 World Congress on Computer Science and Information Engineering Vol.1 (2009) pp 44-48.

[14] "T. Camilo et al", "Mobility management in IP- based Wireless Sensor Networks" World of Wireless, Mobile and Multimedia Networks. International Symposium on, Vol.23.No.26 (2008) pp1-8.

[15] "V. Devarapalli et al", "Network Mobility (NEMO) Basic Support Protocol" RFC-3963, January 2005.
[16] "R. Wakikawa et al", "Problem Statement and Requirements for MANEMO" Internet draft (2007) pp $1-23$.

[17] "Na Li et al", "Privacy preservation in wireless sensor networks: A state-of-the-art survey" Ad Hoc Networks 7 (2009) pp 1501-1514.

[18] "K. Lorincz et al", "Sensor networks for emergency response: challenges and opportunities" Pervasive computing, Vol.12 (2004) pp 16-22.

[19] "A. Wood et al", "ALARM-NET: Wireless sensor networks for assisted-living and residential monitoring" SiteSeerX (2006) pp 1-14.

[20] "U. Anliker et al”,"AMON: A Wearable Multi-parameter Medical Monitoring and Alert System" IEEE Transactions on Information Technology in Biomedicine Vol.8.No.4 (2004) pp 415- 427.

[21] "Dario Iafusco et al", "Usefulness or Uselessness of GlucoWatch in Monitoring Hypoglycemia in Children and Adolescents" PEDIATRICS Vol.113.No.1 (2004) pp 175-176.

[22] "I. F. Akyildiz et al", "Underwater acoustic sensor networks: research challenges" Ad Hoc Networks 3 (2005) pp 257-279.

[23] "M.C. Domingo et al", "Energy analysis of routing protocols for underwater wireless sensor networks" Computer Communications. Vol.31.No.6 (2008) pp 1227-1238.

[24] "Guang-Zhong Yang", "Cognitive sensing for body sensor networks" Sensors, 2008 IEEE (2008) pp 26-29.

[25] "G. Zhou et al", "SAS: Self-Adaptive Spectrum Management for Wireless Sensor Networks" Computer Communications and Networks, 2009. ICCCN 2009. Proceedings of 18th International Conference on, Vol.3.No.6 (2009) pp 1-6.

[26] "A. Liutkevicius et al", "A Survey of Wireless Sensor Network Interconnection to External Networks" Novel Algorithms and Techniques in Telecommunications and Networking, (2010) pp 41-46.

[27] "AJG Sanchez et al","Wireless Sensor Network Deployment for Monitoring Wildlife Passages Sensors", Vol.10.No.8 (2010) pp 7236-7262.

[28] "Y. Zeng et al", "An Emergency-Adaptive Routing Scheme for Wireless Sensor Networks for Building Fire Hazard Monitoring Sensors", Vol.10.No.6 (2010) pp 6128-6148.

[29] "A. Zafeiropoulos et al","NEURON: Enabling Autonomicity in Wirless Sensor Networks Sensors", Vol.10.No.5 (2010) pp 5233- 5262.

[30] "M. Alaei et al","A Method for Clustering and Cooperation in Wireless Multimedia Sensor Networks Sensors", Vol.10.No.4 (2010) pp 3145-3169.

[31] "L. Schor et al", "Towards a zero-configuration wireless sensor network architecture for smart buildings" Conference on Embedded Networked Sensor Systems archive (2009) pp 31-36.

[32] "L. Liu", "Research on Environment-Adaptive 
Architecture Model of Wireless Sensor Networks" Second International Conference on Networks Security, Wireless Communications and Trusted Computing (2010) pp 130-133.

[33] "C. Lin et al", "A Distributed Efficient Architecture for Wireless Sensor Networks" 21st International Conference on Advanced Information Networking and Applications Workshops Vol.2 (2007) pp 429-434.
[34] "J. Lukkien et al", "The WASP Architecture for Wireless Sensor Networks" Communications in Computer and Information Science Vol.11 (2008) pp 430-447.

[35] 'I. F. Khan et al”, “A Survey on Routing Protocols and Problem of Holes in Wireless Sensor Networks" In proceeding International Conference on Advanced 